\title{
Differential expression of factor XIIla and CD34 in cutaneous mesenchymal tumors
}

The histogenetic relationship amongst various dendritic cells of the dermis which may express markers including factor XIIIa (FXIIIa) or CD34 remains unclear. In this study we utilized a sensitive indirect immunoperoxidase staining technique to identify CD34 and FXIIIa, as well the monocyte/macrophage markers KP-1 and MAC 387 expression in a variety of cutaneous dermal tumors of mesenchymal origin to see if differential expression of CD34 vs FXIIIa exists. Tumors studied included dermatofibroma (DF) $(\mathrm{N}=10)$, keloid $(\mathrm{N}=9)$, atypical fibroxanthoma $(\mathrm{AFX})(\mathrm{N}=3)$, and dermatofibrosarcoma protuberans (DFSP) $(\mathrm{N}=7)$. DF were all composed of FXIIIa + spindleshaped and stellate tumor cells (mean score $=4.9$ or $\geq 75 \%$ FXIIIa + ) as previously reported, but these cells rarely $(<10 \%)$ expressed CD34. Six of 7 DFSP were found to be $>75 \%$ CD34+ and FXIIIa negative, while one DFSP was negative for both CD34 and FXIIIa. In all DFSP, there were trapped FXIIIa + cells which were distinct from the spindle-shaped tumor cells. AFX showed sparse populations of FXIIIa + cells in the stroma (mean score $=1.33$ or $10-25 \%$ positive), which were distinct from the atypical giant cells characteristic of these tumors. Keloid similarly contained trapped FXIIIa + cells (mean score $=0.44$ or $<5 \%$ positive) that were distinct from the spindle-shaped fibroblasts of the tumor mass. Dendritic and spindle-shaped cells within these tumors were consistently both KP-1 and Mac-387 negative, while all lesions studied were characterized by scattered round, histiocytic cells which were KP-1+ and/or Mac-387+ irrespective of tumor cell type. We suggest that these tumors can be delineated by their relative degrees of FXIIIa and CD34 expression and that these neoplasms may be a useful link with which to study the relationship between CD $34+$ cells and dermal dendrocytes.

Altman DA, Nickoloff BJ, Fivenson DP. Differential expression of factor XIIIa and CD34 in cutaneous mesenchymal tumors. J Cutan Pathol 1993: 20: 154-158. (C) Munksgaard 1993.

\section{David A. Altman', Brian J. Nickoloff ${ }^{2}$, David P. Fivenson ${ }^{1}$}

Departments of 'Dermatology, Henry Ford Hospital, Detroit, ${ }^{2}$ Pathology, University of Michigan Medical Center, Ann Arbor, USA

David A. Altman, 2799 W. Grand Blvd. Detroit, MI 48202, USA

Accepted August 4, 1992
Recent work has demonstrated the presence of populations of dendritic cells in the dermis. One subset of these cells are thought to be of bone marrow origin and are known as dermal dendrocytes (DD). They express factor XIIIa (FXIIIa) and

Supported in part by NIH grants AR31857, AR40065, AR01823, AR40488 (BJN). have been shown to compose certain cutaneous tumors such as dermatofibromas (1). FXIIIa + DD may be phagocytic and have been proposed to be antigen presenting cells (APC) distinct from Langerhans cells and other APC of the skin $(2,3)$. A similar population of dendritic cells, which express the antigen CD34, has also recently been identified in human reticular dermis, often in coexistence with FXIIIa + DD (4). CD34 has been described as a 
Table 1. Mesenchymal tumor expression of factor XIIIa and CD34

\begin{tabular}{|c|c|c|}
\hline Tumor type & Factor XIIIa score* & CD34 score* \\
\hline Dermatofibroma & $\begin{array}{l}4.90 \pm 0.10^{\star *} \\
(n=10)\end{array}$ & $\begin{array}{l}0.10 \pm 0.10 \\
(n=10)\end{array}$ \\
\hline Atypical fibroxanthoma & $\begin{array}{l}1.33 \pm 0.34 \\
(n=3)\end{array}$ & $\begin{array}{l}0.00 \pm 0.00 \\
(n=3)\end{array}$ \\
\hline Keloid & $\begin{array}{l}0.44 \pm 0.18 \\
(n=9)\end{array}$ & $\begin{array}{l}0.00 \pm 0.00 \\
(n=9)\end{array}$ \\
\hline $\begin{array}{l}\text { Dermatofibrosarcoma } \\
\text { protuberans }\end{array}$ & $\begin{array}{l}1.0 \pm 0.00 \\
(n=7)\end{array}$ & $\begin{array}{l}4.43 \pm 0.57^{* * *} \\
(n=7)\end{array}$ \\
\hline \multirow{8}{*}{\multicolumn{3}{|c|}{ 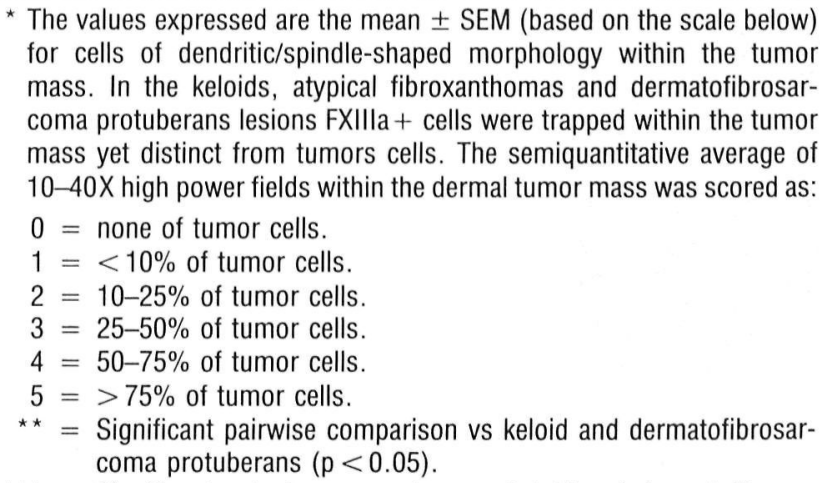 }} \\
\hline & & \\
\hline & & \\
\hline & & \\
\hline & & \\
\hline & & \\
\hline & & \\
\hline & & \\
\hline $\begin{aligned} * * * & =\text { Significant pair } \\
& (p<0.05) .\end{aligned}$ & & dermatofibroma \\
\hline
\end{tabular}

hematopoietic progenitor antigen is expressed by $<1 \%$ of bone marrow cells. It is unclear if CD34+ dendritic cells may denote a similar stem cell population in the skin. If so, these cells may serve as a reservoir of mesenchymal precursor cells which could differentiate upon appropriate stimulation into FXIIIa + DD $(3,4)$.

The current study employs a sensitive immunoperoxidase staining technique to identify populations of both CD34+ and FXIIIa + dendritic cells within tumors of mesenchymal origin including, dermatofibromas (DF), atypical fibroxanthoma (AFX), dermatofibrosarcoma protuberans (DFSP), and keloid. We report significant differential expression of FXIIIa and CD34 in these cutaneous tumors.

\section{Material and methods}

\section{Patients}

Subjects were identified by diagnosis from biopsy records on file in the Departments of Pathology at Henry Ford Hospital, and the University of Michigan Medical Center. Included in this study were serial paraffin sections from $10 \mathrm{DF}, 9$ keloids, 3 AFX, and 7 DFSP. Original hematoxylin and eosin slides were reviewed to confirm the recorded diagnoses.

\section{Indirect immunoperoxidase}

Indirect immunoperoxidase (IPX) staining was performed on $4 \mu \mathrm{m}$ paraffin-imbedded sections which were deparaffinized in xylene and dehydrated in ethyl alcohol. Sections were incubated for $40 \mathrm{~min}$ with trypsin $0.1 \%$ (Sigma, St. Louis, MO) in phosphate buffered saline $0.05 \mathrm{M}$ (PBS), with $\mathrm{CaCl}_{2}$ $0.1 \%$. Endogenous peroxidase activity was inhibited with equal proportions of $100 \%$ methanol and $3 \%$ hydrogen peroxide, prior to blockage with horse serum and exposure to primary antibodies for $1 \mathrm{~h}$ at room temperature. Antibodies used were diluted in PBS with $0.5 \%$ bovine serum albumin and included: anti-human FXIIIa (1:400) and FXIIIs (1:400) (Calbiochem, LaJolla, CA), Mac-387 (1:50), KP-1 (1:50) (Dako, Carperteria, CA), and CD34 (1:10) (HPCA-1, Becton Dickinson, Mountainview, CA). Antibodies were visualized using the avidin/biotin peroxidase technique (Vectastain ABC Kit, Vector Labs Inc, Burlingame, CA). The chromagen was 3-amino, 9-ethyl carbazole (AEC-Sigma, St. Louis, MO). All post-fixation procedures were performed at room temperature. An irrelevant, isotype-matched antibody was included in each experiment as a control.

Dendritic and spindle-shaped dermal cells from within these tumors with positive staining by the IPX technique were evaluated semiquantitatively by scoring the average number positive cells within the dermal tumor mass in 10 high-power fields (40X), employing the following scoring system: $0=$ no dendritic/spindle-shaped cell staining, $1=$ $<10 \%$ of dendritic/spindle-shaped cell staining, $2=10-25 \%$ of dendritic/spindle-shaped cell staining, $3=25-50 \%$ of dendritic/spindle-shaped cell staining, $4=50-75 \%$ of dendritic/spindle-shaped cell staining, $5=>75 \%$ of dendritic/spindleshaped cell staining. Only those cells with visible nuclei and discernable processes were included in this analysis.

\section{Statistical analysis}

A non-parametric analysis of variance (KruskalWallis test) was first performed for the mean FXIII a and CD34 expression within each disease group. To study FXIIIa and CD34 staining among the DF, keloid, AFX, and DFSP groups, pairwise comparisons were used with Bonferoni's correction for multiple comparisons.

\section{Results}

The results are summarized in Table 1. All DF $(\mathrm{N}=10)$ studied expressed FXIIIa on the majority of spindle-shaped and stellate tumor cells (Fig. la). The average score $( \pm$ SEM) of DF tumor cell expression of FXIIIa was $4.90 \pm 0.10$. CD34 staining of these tumor cells was rare, and CD $34+$ cells were limited to the vascular endothelium and a margin of 


\section{Altman et al.}
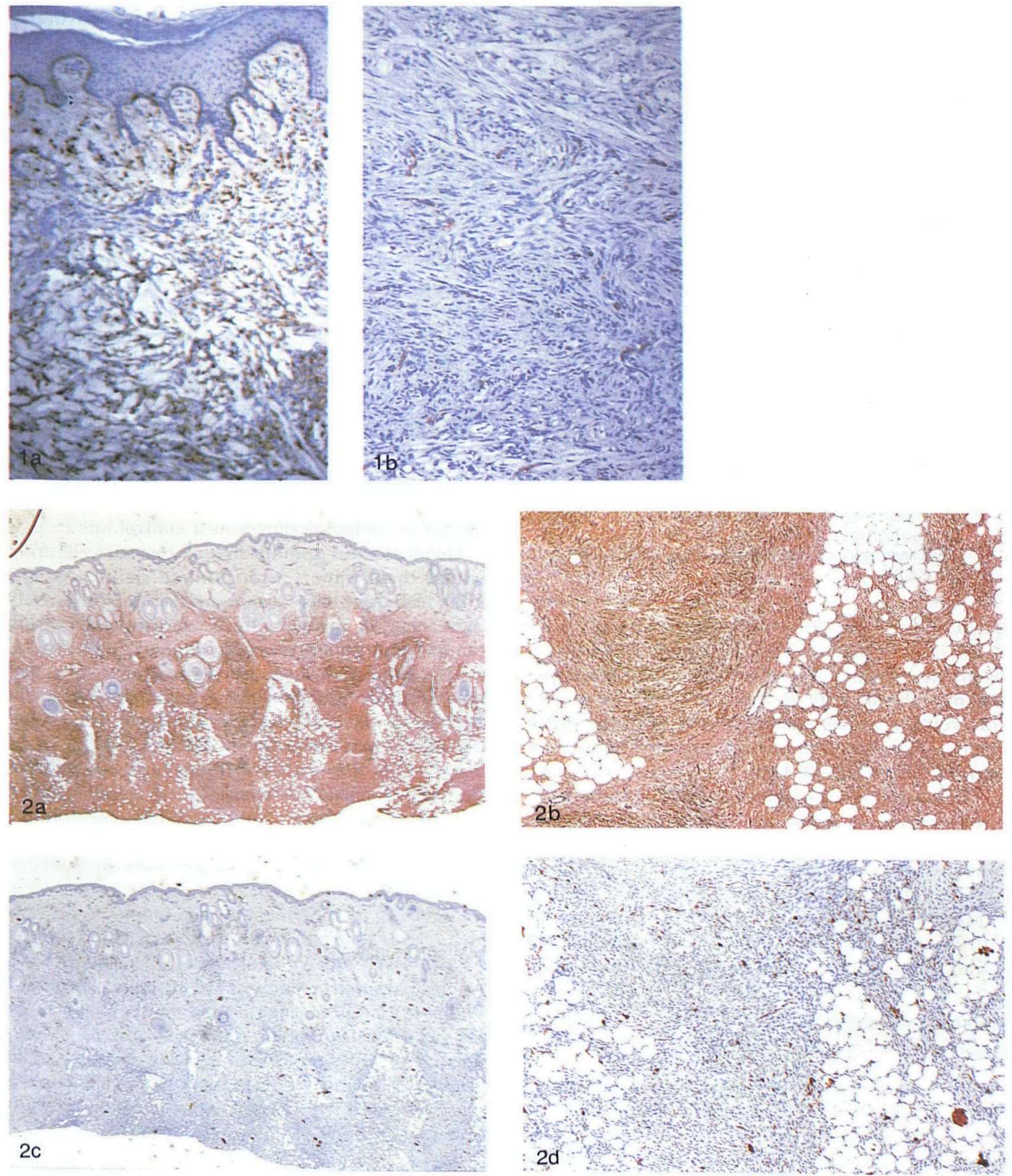

Fig. 1. Demonstration of Factor XIIIa + dendritic cells in dermatofibroma by immunoperoxidase staining (Fig. 1A, $\times 10)$ and CD34+ cells limited the vessels in the same lesion (Fig. 1B, × 50).

Fig. 2. Demonstration of the universal expression of CD34 by spindle-shaped tumors cells in dermatofibrosarcoma protuberans by immunoperoxidase staining (Fig. $2 \mathrm{~A}, \times 5,2 \mathrm{~B}, \times 25)$ and the sparce number of FXIIIa + cells scattered throughout the same lesion (Fig. 2C, $\times 5,2 \mathrm{D}, \times 25)$. Note how CD34 allows demonstration of the deeply invasive aspects of the tumor in the subcutancous fat. 
dendritic cells in the reticular dermis with similar morphology as previously described (Fig. 1b) (4).

Many of the stromal cells in $\operatorname{AFX}(\mathrm{N}=3)$ were dendritic and some expressed FXIIIa (mean score $=1.3 \pm 0.35)$. CD34 staining was rarely seen on any of these dendritic cells. In none of the AFX were $>25 \%$ of lesional cells FXIIIa + and these cells composed a separate population of supporting cells, readily distinguished from the atypical giant cells and foam cells characteristic of AFX.

Four of 9 keloids also contained scattered FXI$\mathrm{IIa}+$ dendritic cells in the tumor mass. These cells were morphologically distinct from the spindleshaped fibroblasts in the keloid and appeared as if trapped within the tumor mass. CD34 expression in a keloid was limited to the vascular endothelium.

CD34 consistently stained all the large, atypical, spindle-shaped tumor cells within 6/7 DFSP, whereas one case was repeatedly CD34 negative. The mean CD34 score for the 7 cases was $4.43 \pm 0.57$. CD 34 delineated the deeper, more invasive aspects of the tumors (Figs 2a,b). All DFSP studied showed scattered FXIIIa + small dendritic cells of typical DD morphology (Figs 2c,d). The mean score for FXIIIa expression by the 7 DFSP studied was 1.0, consistent with this scattering of normal appearing DD trapped within the lesions.

Similar to prior reports, we found Mac-387 and KP-1 did not identify DD in paraffin sections $(2,5)$. In the tumors studied, Mac-387 and KP-1 stained scattered dermal cells characterized by large histiocytic cells, pale nuclei, abundant cytoplasm and a lack of distinct dendritic processes.

Statistically significant increases in FXIIIa+ cells were shown for the DF group as compared to the keloids and DFSP $(p<0.05)$ CD $34+$ cells were significantly increased in the DFSP group as compared to the DF and keloid groups $(p<0.05)$. Comparisons of AFX vs DFSP and AFX vs DF were not statistically relevant due to the small numbers of cases.

\section{Discussion}

Dendritic cells of the dermis have recently been the focus of intensive work. Cerio et al. defined dermal dendrocytes as a population of dendritic cells in the upper dermis of normal skin which express FXIIIa as well as HLA-DR (5). These cells also express CD14, CD45, and CD54, suggesting a mononuclear cell origin for DD (2). In normal human skin, FXIIIa + DD are located perivascularly and limited to the papillary and upper reticular dermis. A morphologically similar, CD 34 +/FXIIIa negative population of dendritic cells has been recently identified and is concentrated immediately below the FXIIIa + DD in the deep reticular dermis $(3,4)$.
CD34 is a monomeric 115-kd glycoprotein expressed on hematopoietic progenitor cells in bone marrow as well as on endothelial cells in the skin and a wide variety of vascular and spindle-shaped cell tumors $(4,6-8)$.

FXIIIa and CD34 are both reported to be expressed by populations of spindle-shaped tumor cells within Kaposi's sarcoma (KS) (4, 9, 10). CD34 and FXIII co-expression by various proportions of spindle-shaped cells within KS lesions has led to speculation of a histogenetic connection between these two cell populations. KS cells were previously felt to be a neoplastic proliferation of endothelial cells alone, but have been recently suggested to be related to DD as well (9).

Although the sample sizes studied were not large, the current study demonstrates that differential expression of CD34 and FXIIIa by dendritic cells within benign and malignant mesenchymal dermal tumors is more regularly seen than co-expression, and may prove to be a useful method to delineate these lesions histologically. Similar to recent reports, we found the spindle-shaped cells in 6/7 DFSP to be CD34+ and FXIIIa negative $(8,9)$. Our finding in DF and keloid demonstrated that there are distinct FXIIIa/CD34 profiles for each of these benign tumors as well. If supported by larger studies, differential FXIIIa vs CD34 expression in these tumors may become a useful immunohistochemical adjunct in histologic delineation as in general: DF are FXIIIa + and CD34 negative, DFSP are FXIIIa negative and CD34+, and keloids are both FXIIIa and CD34 negative.

\section{References}

1. Cerio R, Spaull J, Wilson Jones E. Histiocytoma cutis, a tumor of dermal dendrocytes. BrJ Dermatol 1989: 120: 197.

2. Cerio R, Griffiths CEM, Cooper KD, Nickoloff BJ, Headington JT. Characterization of factor XIIIa positive dermal dendrocytes in normal and inflamed skin. Br J Dermatol 1989: 121: 421 .

3. Altman DA, Fivenson DP, Lee MW. Minocycline hyperpigmentation as a model for in-situ phagocytic activity of factor XIIIa positive dermal dendrocytes. J Cutan Pathol (in press).

4. Nickoloff BJ. The human progenitor antigen (CD34) is localized on endothelial cells, dermal dendritic cells, and perifollicular cells in formalin fixed normal skin, and on proliferating endothelial cells and stromal spindle-shaped cells in Kaposi's sarcoma. Arch Dermatol 1991: 127: 523.

5. Cerio R, Spaull J, Oliver GF, Wilson Jones E. A study of factor XIIIa and MAC 387 immunolabeling in normal and pathologic skin. Am J Dermatopathol 1990: 12(3): 221.

6. Traweek ST, Kandalaft PL, Mehta P, Battifora H. The human hematopoietic progenitor cell antigen (CD34) in vascular neoplasia. Am J Clin Pathol 1991: 96: 25.

7. Gohen PR, Rapini RP, Farhood A. The expression of the human hermatopoietic progenitor cell antigen CD34 in vas- 


\section{Altman et al.}

cular and spindled cell tumors. J Cutan Pathol 1991: 18: $363 \Lambda$.

8. Aiba S, Tabata N, Ishii H, Ootani H, Tagami H. Dermatofibrosarcoma protuberans is a unique fibrohistiocytic tumour expressing CD34. Br J Dermatol 1992: 127: 79.
9. Nickoloff BJ, Griffiths CEM. Factor XIIIa-expressing dermal dendrocytes in AIDS-associated cutancous Kaposi's sarcomas. Science 1989: 243: 1736.

10. Nickoloff BJ, Griffiths CEM. The spindle shaped cells in cutaneous Kaposi's sarcoma. Am J Pathol 1989: 135: 793. 
This document is a scanned copy of a printed document. No warranty is given about the accuracy of the copy. Users should refer to the original published version of the material. 